\title{
Construcción y Validación de un Instrumento para Evaluar el Consumo de Alimentos con Rojo Allura
}

\author{
Rebeca Dey ${ }^{(1)}$, Gladys Linares ${ }^{(1)}$, Ricardo Munguía $^{(1,2)}$ y Edith Chávez ${ }^{(1,2) *}$ \\ (1) BUAP Posgrado en Ciencias Ambientales-ICUAP, Edificio IC6 Ciudad Universitaria \\ (2) Centro de Investigaciones en Ciencias Microbiológicas del Instituto de Ciencias BUAP, Edificio IC11 \\ Ciudad Universitaria, Puebla, México. (e-mail: edith.chavez@correo.buap.mx) \\ ${ }^{*}$ Autor a quien debe ser dirigida la correspondencia.
}

Recibido Ago. 22, 2018; Aceptado Nov. 19, 2018; Versión final Ene. 22, 2019, Publicado Jun. 2019

\begin{abstract}
Resumen
Se realizó un estudio exploratorio para identificar los alimentos procesados que contienen rojo allura y que sean expendidos cerca de escuelas de educación básica en la Ciudad de Puebla-México. Hay estudios que confirman la toxicidad del colorante rojo allura y su posible relacion con la generación de metabolitos carcinogénicos. Para el estudio, se diseñó un instrumento de medición para determinar la frecuencia de consumo en niños de 10 a 13 años de edad. Se inició con un análisis descriptivo mediante un estudio de campo y con los productos identificados se diseñó el instrumento. Posteriormente se validó con el coeficiente del alfa de Cronbach. Se determinó que la población infantil consume frecuentemente alimentos procesados con rojo allura, teniendo como consumo diario las botanas (frituras), seguido por cereales y yogurt. El estudio logró diseñar y validar un instrumento de confiabilidad, que permitió medir la frecuencia de consumo de alimentos procesados con rojo allura en infantes.
\end{abstract}

Palabras clave: color en alimentos; rojo allura; alfa de Conbrach; agentes cancerígenos

\section{Construction and Validation of an Instrument for Evaluating the Consumption of Food with Red Allura}

\begin{abstract}
An exploratory study was conducted to identify processed foods that contain allura red and are sold near primary schools in the City of Puebla, Mexico. There are studies that confirm the toxicity of allura red and its probable relation with the generation of cancer metabolites. For the study, a measuring instrument was designed to determine the frequency of consumption in children from 10 to 13 years of age. For this, the instrument was designed following a descriptive analysis by means of a field study identifying common commercial products. Later the Cronbach's alpha coefficient was used to validate the statistical reliability of the instrument. It was determined that the infant population oftenly consumes foods processed with red allura, having as daily consumption snacks (fried foods), followed by cereals and yogurt. This study was able to design and validate a reliable instrument, which allows to measure the frequency of consumption of foods processed with red allura in infants.
\end{abstract}

Keywords: food color; red allura; Cronbach's alpha; cancer agents 


\section{INTRODUCCIÓN}

El color en los alimentos procesados es una de las propiedades imprescindibles para su evaluación, tiene la finalidad de volverlo atractivo y apetitoso al consumidor. Los colorantes sintéticos son ampliamente utilizados en la industria alimentaria por su estabilidad y bajo costo, los más empleados son los azoicos con un $65 \%$ en el mercado, ofrecen colores vivos y resisten diversas condiciones del proceso alimentario (Burrows A., 2009; Yamjala et al., 2016). El rojo allura conocido también como rojo 40 con número $E$ 129, es un colorante alimentario sintético de naturaleza azo (-N=N-), con una Ingesta Diaria Admisible (IDA) de $7 \mathrm{mg} / \mathrm{kg}$ de peso corporal por día recomendado por la Autoridad Europea de Seguridad Alimentaria (ESFA por sus siglas en inglés) en 2015. En la actualidad existe una gran gama de productos procesados los cuales contienen dicho colorante como: bebidas, lácteos, productos de panadería, cárnicos, grenetinas, polvos de postre, dulces, cereales, medicamentos, cosméticos, tatuajes y comida para mascotas (Stevens et al., 2014; Yamjala et al., 2016), mucho de ellos están fácilmente al alcance de infantes, ya sea en sus hogares, en los planteles educativos y/o centros comerciales (Monteiro et al., 2017).

El gobierno del Reino Unido exhorta a los demás países europeos a prohibir voluntariamente el uso de colorantes en alimentos procesados por el resguardo de la seguridad alimentaria, debido a que se encuentra implicado en la salud infantil por alergias, hiperactividad, déficit de atención y liberador de histamina intensificando los síntomas de asma en infantes (Gupta y Suhas 2009, Vodjani y Vodjani, 2015), con respecto a la toxicidad del colorante rojo allura, algunos autores confirman que existen daños citológicos (Schimada et al., 2010, Noorasfshan et al., 2018) pero últimamente, el interés incrementa por la participación de la microbiota intestinal en la reducción del enlace azo del colorante generando metabolitos carcinogénicos, aunque esto depende de la frecuente ingesta (Feng et al., 2012), también se le ha puesto atención al sinergismos ejercido por diferentes colorantes que se encuentran en un mismo alimento (Stevens et al., 2015, Amchova et al., 2015).

En México como en otros países, la población no le da interés a la información de los ingredientes registrados en las etiquetas de los productos, además de la nula información proporcionada por los gobiernos en las cantidades permisibles de consumo de los colorantes (Cheung et al., 2016), por lo que el consumidor desconoce la peligrosidad detrás de un color atractivo (Vodjani y Vodjani, 2015). Diversas investigaciones han coincidido que la población infantil es la más vulnerables en ingerir alimentos con colorantes y generar trastornos alérgicos o de reactividad inmunológica (Stevens et al., 2014), debido a que la ingesta diaria admisible se excede significativamente en los alimentos (Dixit et al, 2010, Batada y Jacobson, 2016). En la Ciudad de Puebla-México se desconoce la variedad de alimentos procesados que se distribuyen en los diferentes lugares de expendio y que puedan estar al alcance de la población infantil, también se desconoce la frecuencia de consumo de los alimentos que contienen rojo allura. Por lo antes citado, la presente investigación realizó un estudio de campo de forma aleatoria, recorriendo tiendas de autoservicio, tiendas locales y en cafeterías de escuelas de la Ciudad de Puebla para realizar un listado de los alimentos procesados con rojo allura y con ello construir y validar un instrumento de evaluación para medir la frecuencia de consumo de dichos productos en una población infantil.

\section{METODOLOGÍA}

Se realizó un estudio exploratorio descriptivo de tipo transversal en la Ciudad de Puebla, para conocer los alimentos procesados con rojo allura que están expuestos al consumidor, para ello se recorrieron tiendas de autoservicios, tiendas locales y cafeterías de escuelas de nivel básico; en cada lugar se revisaron las etiquetas de los productos y se registraron aquellos que contienen el colorante. Con base al listado de alimentos se realizó un cuestionario de 25 ítems dirigida a la ingesta de infantes, se utilizó la escala de Likert debido a que ofrece una graduación de la opinión de la frecuencia de consumo en las personas encuestadas, dicha escala consistió en 4 puntos: muy frecuente (consumo diario), frecuente ( 1 a 3 veces a la semana), ocasionalmente (1 vez a la quincena) y nunca (no lo han probado).

El cuestionario se aplicó a 28 personas como prueba piloto, para verificar si las preguntas son entendibles, correctas, comprensibles y adecuadas; si el ordenamiento interno es lógico y el tiempo de aplicación está dentro de lo aceptable. Posteriormente se construyó un instrumento y se utilizó un estadístico de fiabilidad el coeficiente del alfa de Cronbach para medir la consistencia interna entre los ítems y validar la confiabilidad de medición. Para calcular dicho coeficiente, se utilizaron dos métodos: Varianza de ítems representado por la ecuación (1) donde $\alpha=$ Alfa de Cronbach, $\mathrm{k}=$ Número de ítems, $\mathrm{Vi}=$ varianza de cada ítem y $\mathrm{Vt}=\mathrm{varianza}$ del total. También se utilizó la matriz de correlación, ecuación (2) donde $\alpha=$ Alfa de Cronbach, $n=$ Número de ítems y $\mathrm{p}=$ promedio de las correlaciones lineales de cada uno de los ítems. Además de ambos métodos se utilizó el programa estadístico SPSS versión 19. Los valores superiores a 0.7 - 0.8 de alfa de Cronbach se consideraron aceptables o suficientes para validar el instrumento. 


$$
\begin{aligned}
& \alpha=\frac{k}{k-1}\left[1-\frac{\sum V i}{V t}\right] \\
& \alpha=\frac{n p}{1+p(n-1)}
\end{aligned}
$$

Una vez validado el instrumento para medir la frecuencia de consumo de alimentos procesados con rojo allura, se aplicó a madres voluntarias como responsables de la alimentación de sus niños de 10 a 13 años de edad y que cursan el 6 año del nivel básico escolar. Con los datos obtenidos, se realizó un análisis descriptivo con gráficas, tablas de frecuencias y de distribución conjunta, para conocer los alimentos que se consumen con mayor frecuencia en la población infantil.

\section{RESULTADOS Y DISCUSIÓN}

Se realizó un estudio exploratorio transversal descriptivo con una población finita, conformada por 197 madres encuestas que dieron información sobre la alimentación de sus hijos de 10 a 13 años de edad que se encontraban estudiando en el nivel básico de tres escuelas primarias de la Ciudad de Puebla.

\section{Estudio exploratorio}

La gama de productos alimentarios con el colorante rojo 40, se obtuvo de tres tiendas de autoservicios, 4 tiendas abarrotes y 3 cafeterías de escuelas primarias, identificando los productos con el colorante al observar las etiquetas. Las tiendas a las que se visitaron fueron por la cercanía a las escuelas primarias donde se llevó acabo la aplicación del constructo. Durante la revisión de etiquetas, se registraron los nombres de los alimentos procesados con rojo allura en los diferentes lugares de expedición, como se halló una gran variedad de productos con este colorante, se realizaron 5 grupos: bebidas, confitería, lácteos, productos para elaborar comida y otros, para su análisis y presentación, en cada grupo existen productos de diferente marca y de diferente lugar de procedencia (Tabla 1).

Tabla 1: Alimentos con rojo allura, hallados en lugares cercanos a escuelas primarias de la Ciudad de Puebla, 2018

\begin{tabular}{|l|l|}
\hline \multicolumn{1}{|c|}{ Grupo } & \multicolumn{1}{c|}{ Tipos de alimentos procesados } \\
\hline Bebidas & $\begin{array}{l}\text { Refrescos, agua de sabor preparada, jugos, } \\
\text { bebidas hidratantes, polvos para preparar agua de } \\
\text { sabor, saborizantes, jarabes o concentrados de } \\
\text { fresa, uva, arándano, frambuesa y/o Jamaica. }\end{array}$ \\
\hline Confitería & $\begin{array}{l}\text { Cereales, galletas, barras energéticas, pastelitos, } \\
\text { dulces, frituras, botanas saladas. }\end{array}$ \\
\hline Lácteos & Yogurt y leches con sabor a fresa. \\
\hline $\begin{array}{l}\text { Productos para } \\
\text { elaborar comida }\end{array}$ & $\begin{array}{l}\text { Purés de tomate, sazonadores, salsas y gelatinas } \\
\text { de sabor uva, fresa y grosella. }\end{array}$ \\
\hline Otros productos & $\begin{array}{l}\text { Se hace referencia a las avenas instantáneas, } \\
\text { maizenas y harinas para hotcakes. }\end{array}$ \\
\hline
\end{tabular}

En las bebidas se agruparon: refrescos (sodas), agua embotellada con sabor, jugos envasados, bebidas hidratantes, concentrados o pulpas de fruta y sobres de saborizante en polvo para diluir en agua; en el grupo de confitería se encuentran los cereales infantiles, galletas con sabor, barras energéticas con pulpa de fruta, pan o pastelitos embolsados, caramelos, golosinas, frituras y botanas saladas; en los lácteos se encuentran los productos derivados de la leche como yogurt en sus diferentes presentaciones líquido y semisólido, licuados y leche entera de sabor líquida o en polvo; en los productos para elaborar comida también se reporta en su etiqueta la presencia del colorante como: purés instantáneos de tomate para sopa o guisados, sazonadores, salsas picantes y gelatinas; y en el último grupo otros, se encuentra productos a base de fécula de maíz o de trigo para preparar harinas, avenas instantáneas, maizena de sabor y harinas para hotcakes. En todos los productos registrados se encontró una similitud del sabor con la presencia del colorante rojo allura, es decir todos los productos con sabor a frutas, fresa, uva, manzana, arándano y chocolate contienen el colorante, aunque en algunos productos no se asocia como las harinas o semolinas. 
La gran variedad de productos con rojo allura encontrados en este estudio puede estar relacionado con el incremento de certificaciones que cada año se dan en la industria alimentaria para el uso de colorantes en cantidad, tipos o aumentar su aplicación en diversos productos, como es el caso de la Administración de Alimentos y Medicamentos (FDA por sus siglas en ingles) de USA quien aprobó el incremento en las cantidades totales de colorantes como rojo 40 (rojo allura), amarillo 5 y amarillo 6 (Stevens et al., 2014). Batada y col. (2016) en un estudio realizado en Carolina del Norte (USA), reportan que el $43 \%$ de los alimentos dirigidos a niños y que son expedidos en un centro comercial contienen colorantes artificiales, principalmente golosinas, aperitivos con sabor a fruta y las bebidas con más del $89 \%$, siendo el colorante rojo allura el que predomino en los productos. Otra posible respuesta, se relaciona con los cambios de alimentación, donde los productos le proporcionan sabor agradable a base de ingredientes baratos y aditivos. Las empresas transnacionales pueden producir y distribuir los productos en gran medida, para que la población continúe consumiendo refrescos, galletas, dulces, barras, cereales, bebidas carbonatadas azucaradas, harinas y almidones, convirtiéndose en una ventaja pe cápita sin que preocupe la salud del consumidor (Monteiro et al., 2013).

La industria alimentaria de algunos países como Suiza, Bélgica, Francia y Dinamarca, se encuentra estrictamente controlado el uso de colorantes sintéticos y prohibido el uso de rojo allura, debido a los efectos provocados en la salud como: daño cerebral, náuseas, enfermedades cardiacas entre otros (Noorafshan et al., 2017). Sin embargo, en países donde es autorizado el rojo allura, los productores de alimentos procesados, lo utilizan de manera ilimitada o lo combinan con otros colores, dificultado la cantidad exacta que queda en el alimento. En atención a ello, existen diversos métodos químicos y avanzados para monitorear el colorante rojo allura tanto en alimentos como en bebidas, pero implican tiempo y costo (Rovina et al., 2016). Dichos métodos ayudan alertar al consumidor, para que su ingesta diaria del colorante no sea mayor a la recomendada.

Por otro lado, la mayoría de las encuestas realizadas en México, relacionan la ingesta de alimentos con la dieta y la valoración de nutrición en niños, no existe un estudio específico sobre el consumo de productos con rojo 40. Algunos países como Italia, han realizado la evaluación de la exposición del colorante rojo allura al consumir refrescos y jugos, dicha información fue recopilada de encuestas ya establecidas por la INRANSCAI 2005-06 así como datos de ventas anuales de 27 tiendas seleccionadas (Fallico et al., 2011).

\section{Construcción del instrumento}

Después de conocer los alimentos procesados con rojo allura que se distribuyen en la Ciudad de Puebla, se realizó un cuestionario con 25 preguntas (ítems) y se aplicó a una muestra piloto de 28 encuestados, que permitió hacer las modificaciones pertinentes en las preguntas y con ello construir el instrumento con 21 ítems. En dicha construcción se consideró: los productos registrados en el estudio exploratorio, preguntas relacionadas con el consumo de alimentos con sabor a frutas, fresa, manzana, uva, arándano y chocolate por su relación con el colorante, se utilizó la escala de Likert indicada en la metodología para el análisis de frecuencia de consumo y finalmente se validó el instrumento con el alfa de Conbrach (Tabla 2). Dicho instrumento permitió conocer los alimentos procesados afines al consumo de los infantes.

Tabla 2: Instrumento utilizado y validado, para medir la frecuencia de consumo de alimentos procesados con rojo allura en niños de 10 a 13años en la Ciudad de Puebla en el 2018

Nombre

Edad

\begin{tabular}{|c|c|c|c|c|}
\hline \multirow{3}{*}{$\begin{array}{l}\text { Consumo de alimentos procesados } \\
\text { Los productos que se mencionan pueden ser de diferente marca }\end{array}$} & \multicolumn{4}{|c|}{ Ingesta } \\
\hline & \multicolumn{4}{|c|}{$\begin{array}{l}\text { Marque la casilla que considere más } \\
\text { acorde }\end{array}$} \\
\hline & $\begin{array}{c}\text { Muy } \\
\text { frecuente }\end{array}$ & Frecuente & Ocasional & INunca \\
\hline \multicolumn{5}{|l|}{$\begin{array}{l}\text { 1.-¿Consume refrescos de color rojo sabor tutifruti, arándano, sangría, uva, } \\
\text { manzana? }\end{array}$} \\
\hline \multicolumn{5}{|l|}{ 2.- ¿Consume agua embotellada preparada sabor jamaica o manzana? } \\
\hline \multicolumn{5}{|l|}{ 3.- ¿Consume jugos envasados sabor fresa, uva, manzana, arándano? } \\
\hline 4.-¿Consume bebidas hidratantes de sabor fresa, naranja color rojo? & & & & \\
\hline
\end{tabular}


Tabla 2 (continuación)

5.- ¿Consume y/o se prepara en casa agua con saborizantes (sobre en polvo) de sabor frutas, fresa, uva o manzana?

6.-¿Consume cereales de caja?

7.-¿Consume galletas de chocolate, con bombón, con relleno sabor fresa?.

8.-¿Consume barras energéticas: de fresa, ciruela pasa o de cereales?.

9.-¿Consume pastelitos empaquetados sabor fresa y/o chocolate?

10.- ¿Consume paletas de dulce o de hielo de color sabor grosella o fresa?

11.-¿Consume dulces en forma de gomita sabor fresa?

12.- ¿Consume gelatinas de sabor fresa y/o grosella?

13.-¿Consume caramelos de sabor fresa o color rojo?

14.-¿Consume cacahuates y/o malvaviscos cubiertos de chocolate?

15.-¿Consume paletas o caramelos cubiertos con polvo de chile?

16.-¿Consume frituras embolsadas de papa o de fécula de maíz, con queso o chile?

17.-¿Consume cacahuates con chile?

18.- ¿Consume lácteos o yogurt de sabor fresa, uva y/o arándanos?

19.-¿Consume puré de tomate envasado?

20.-¿Consume salsas picantes envasadas de color rojo?

21.- ¿Consume avena y harinas para hot cakes?

\section{Validación del instrumento}

En el análisis de fiabilidad del instrumento, los valores de alfa de Cronbach mediante la consistencia interna de los ítems y mediante la matriz de correlación fueron de 0.85 y 0.82 respectivamente, lo que representa una buena correlación entre los ítems y escasa variabilidad debida a los consumidores (Tabla 3), dichos valores se compararon con el programa estadístico SPSS (por sus siglas en inglés: Startical Product and Service Solutions), cuyo valor fue superior al 0.80 lo que indica una buena consistencia interna para este instrumento. La validación del instrumento determinó que es adecuado para la determinar la frecuencia de consumo de alimentos procesados con rojo allura en la población de infantil; es válido debido a que la frecuencia de consumo se basó en escala de Likert que ofrece una graduación de la opinión de las personas encuestadas, evitando algún posible sesgo de la respuesta; es fiable ya que es preciso el empleo del mismo y los resultados son veraces y constantes; es sensible debido a que puede ser respondido por otros individuos a través del tiempo y finalmente delimita claramente sus dimensiones de los productos alimentarios con rojo allura.

Tabla 3: Estadísticos de consistencia interna y fiabilidad obtenidos del instrumento de evaluación, para la frecuencia de consumo de los alimentos procesados con rojo allura en la Ciudad de Puebla, 2018

\begin{tabular}{|c|c|c|c|}
\hline \multicolumn{2}{|c|}{ Valores de alfa de Cronbach $(\alpha)$} & $\begin{array}{c}\text { Número de } \\
\text { Elementos }\end{array}$ \\
\cline { 1 - 2 } $\begin{array}{c}\text { Varianza de los } \\
\text { ítems }\end{array}$ & $\begin{array}{c}\text { Matriz de } \\
\text { correlación }\end{array}$ & $\begin{array}{c}\text { SPSS } \\
\text { Versión 19 }\end{array}$ & \multirow{2}{*}{21} \\
\cline { 1 - 3 }$\alpha=0.8571$ & $\alpha=0.822$ & $\alpha=0.857$ & \\
\hline
\end{tabular}

Existen diversos autores que proponen métodos de frecuencia de consumo alimentario, aplicando cuestionarios en los estudios epidemiológicos donde relacionan la ingesta dietética y la enfermedad o factores de riesgo. Los componentes principales de los cuestionarios son la lista de alimentos, la frecuencia de consumo y el tamaño de ración consumida que han favorecido estudios epidemiológicos (Pérez, 2015). Sin 
embargo, para medir la frecuencia de consumo de alimentos con un aditivo en específico no son suficientes, por lo que, en este estudio se propone un instrumento validado con la certeza que medirá la variable que se pretende medir.

\section{Aplicación del Instrumento de medición}

El instrumento validado se aplicó a 197 mamás voluntarias para participar en la encuesta y que tienen niños de 10 a 13 años de edad, en el análisis de datos se hallaron los productos que ingieren los infantes de manera muy frecuente, teniendo en primer lugar las frituras con un $12 \%$, seguido por cereales y el yogur con un 11 y $10 \%$ respectivamente, de acuerdo a la medida Likert la frecuencia corresponde a un consumo diario de dichos alimentos con rojo allura (tabla 2). Dicho resultado concuerda con Doell y col. (2016) quienes reportan una alta exposición del colorante rojo allura y del amarillo 5 en los niños y adolescentes, debido al consumo frecuente de cereales, bebidas (refrescos) y lácteos (yogurt), alimentos que se registraron y analizaron al realizar las encuestas de consumo.

Con respecto al consumo frecuente de alimentos que corresponde a una ingesta de 1 a 3 veces por semana, los infantes prefieren nuevamente las frituras, el yogurt y los cereales con un $44 \%, 39 \%$ y $33 \%$ respectivamente, seguidos por galletas (32\%), jugos envasados (30\%), refrescos (sodas) $(29 \%)$, gelatinas (26\%) y los alimentos con menor porcentaje fue el agua preparada con saborizantes en polvo sabor a fresa y manzana (tabla 4). Los resultados de frecuencia de consumo ocasionalmente (1 vez a la quincena) y nunca (no lo han probado) no se presentan en la tabla 4 debido a que su elección fue mínima y se pretende destacar la frecuencia de consumo diario y/ó de 1 a 3 veces de alimentos procesados.

Tabla 4: Frecuencia de consumo de alimentos procesados con rojo allura, de los niños estudiados en la Ciudad de Puebla, 2018

\begin{tabular}{|l|c|c|}
\hline \multirow{2}{*}{$\begin{array}{c}\text { Alimento } \\
\text { procesado }\end{array}$} & \multicolumn{2}{|c|}{\begin{tabular}{c} 
\% de ingesta \\
\cline { 2 - 3 }
\end{tabular}} \\
\cline { 2 - 3 } & $\begin{array}{c}\text { Muy Frecuente } \\
\text { (Diario) }\end{array}$ & $\begin{array}{c}\text { Frecuente } \\
\text { (1 a 3 veces/sem) } \\
\% \text { (No. de niños) }\end{array}$ \\
\hline Refresco & $4 \%(8)$ & $29 \%(57)$ \\
\hline Agua de sabor & $5 \%(10)$ & $21 \%(41)$ \\
\hline Jugos & $5 \%(10)$ & $30 \%(59)$ \\
\hline Cereales & $11 \%(21)$ & $33 \%(64)$ \\
\hline Galletas & $4 \%(8)$ & $32 \%(63)$ \\
\hline Dulces gomas & $2 \%(4)$ & $24 \%(47)$ \\
\hline Frituras & $12 \%(23)$ & $44 \%(87)$ \\
\hline Yogurt & $10 \%(19)$ & $39 \%(67)$ \\
\hline Purés & $1 \%(2)$ & $25 \%(49)$ \\
\hline Salsas & $1 \%(2)$ & $26 \%(51)$ \\
\hline Gelatina & $0.5 \%(1)$ & $26 \%(51)$ \\
\hline
\end{tabular}

Stevens y col. (2014) reportaron que a partir de una encuesta de evaluación nutricional los niños de 6 a12 años consumen en promedio $517 \mathrm{ml} / \mathrm{d}$ de bebidas endulzadas, el $77 \%$ de los niños diariamente consumen refrescos, el $12 \%$ consume bebidas hidratantes (deportivas) y el $16 \%$ consume bebidas de dieta. Al analizar las bebidas encontraron diversos colorantes como azul brillante, azul 1, rojo allura, amarillo 5 y amarillo 6 siendo el colorante rojo allura el que predomino en los productos. En este estudio también se encontró este colorante en bebidas, dulces y botanas, pero con el constructo reveló que las botanas específicamente las frituras, son el producto con mayor afinidad para su consumo con un $56 \%$, es decir más de mitad de la población infantil estudiada come diariamente frituras o al menos 1 a 3 veces por semana. Se analizó de forma conjunta los productos de mayor consumo por la población infantil: cereales, frituras y yogurt y se encontró que 33 niños prefieren consumir cereales y yogurt, al menos 1 a 3 veces por semana y 27 niños una vez por quincena (tabla 5); en el consumo conjunto de yogurt y frituras, 32 niños consumen 1 a 3 veces por semana y 25 una vez por quincena (tabla 6 ).

Tabla 5: Distribución conjunta del consumo de yogurt y cereales, de los niños estudiados en la Ciudad de Puebla, 2018

\begin{tabular}{|c|c|c|c|c|c|c|}
\hline \multicolumn{7}{|c|}{ Yogurt } \\
\hline \multirow{2}{*}{ Cereales } & & Diario & 1 a 3 veces /semana & $\begin{array}{c}1 \mathrm{vez} \\
\text { /quincena }\end{array}$ & Nunca & Totales \\
\hline & Diario & $6(3 \%)$ & $11(5.5 \%)$ & $4(2 \%)$ & $0(0 \%)$ & $21(10.6 \%)$ \\
\hline
\end{tabular}


Tabla 5 (continuación)

\begin{tabular}{|l|l|c|c|c|c|c|}
\hline & $\begin{array}{l}\text { 1 a 3 veces/ } \\
\text { semana }\end{array}$ & $7(3.5 \%)$ & $33(16.7 \%)$ & $19(9.6 \%)$ & $5(2.5 \%)$ & $64(32.4 \%)$ \\
\cline { 2 - 6 } & $\begin{array}{l}\text { Una vez } \\
\text { /quincena }\end{array}$ & $2(1 \%)$ & $16(8.1 \%)$ & $27(13.7 \%)$ & $9(4.5 \%)$ & $54(27.4 \%)$ \\
\cline { 2 - 7 } & Nunca & $4(2 \%)$ & $17(8.6 \%)$ & $20(10.1 \%)$ & $17(8.6 \%)$ & $58(29.4 \%)$ \\
\cline { 2 - 7 } & Totales & $19(9.6 \%)$ & $77(39 \%)$ & $70(35.5 \%)$ & $31(15.7 \%)$ & $197(100 \%)$ \\
\hline
\end{tabular}

Tabla 6: Distribución conjunta de consumo de yogurt y frituras, de los niños estudiados en la Ciudad de Puebla, 2018

\begin{tabular}{|c|c|c|c|c|c|c|}
\hline \multicolumn{7}{|c|}{ Yogurt } \\
\hline \multirow{6}{*}{ Frituras } & & Diario & 1 a 3 veces /semana & $1 \mathrm{vez} /$ quincena & Nunca & Totales \\
\hline & Diario & $4(2 \%)$ & $11(5.5 \%)$ & $4(2 \%)$ & $4(2 \%)$ & $23(11.6 \%)$ \\
\hline & $\begin{array}{l}\text { a } 3 \\
\text { veces } \\
\text { /semana }\end{array}$ & $10(5 \%)$ & $32(16.2 \%)$ & $31(15.7 \%)$ & $14(7.1 \%)$ & $87(44.1 \%)$ \\
\hline & $\begin{array}{l}\text { Una vez } \\
\text { /quincena }\end{array}$ & $4(2 \%)$ & $30(15.2 \%)$ & $25(12.6 \%)$ & $12(6 \%)$ & $71(36 \%)$ \\
\hline & Nunca & $1(0.5 \%)$ & $4(2 \%)$ & $10(5 \%)$ & $1(0.5 \%)$ & $16(8.1 \%)$ \\
\hline & Totales & $19(9.6 \%)$ & $77(39 \%)$ & $70(35.5 \%)$ & $31(15.7 \%)$ & $197(100 \%)$ \\
\hline
\end{tabular}

Los resultados de este estudio revelan una gran variedad y distribución de alimentos con rojo allura, la población infantil estudiada tiene mayor preferencia en el consumo de frituras ya que este tipo de alimento es una mezcla de contenido energético considerable, escasa en fibras, proteínas, vitaminas y minerales; su consumo diario provoca un desequilibrio nutrimental y contribuye a la obesidad si no existe actividad física (Gharachorlo, 2010). Con respecto al consumo de alimentos con colorantes se ha encontrado una relación con afecciones en la salud, como es el caso de la hiperactividad relacionado con la ingesta de alimentos con el colorante: azul 1, azul 2, rojo allura, amarillo 5 y/o amarillo 6 (Nigg et al., 2012, Ceyhan et al., 2013). En este estudio se halló una frecuencia de consumo de 2 ó 3 alimentos conjuntos con el aditivo, resultado que podría preocupar a corto o largo plazo por los efectos o riesgos a la salud infantil. Martinis y col. (2016) mencionan que las alergias, los efectos conductuales y neurocognitivos relacionadas con el consumo de alimentos con colorantes se ha incrementado en las últimas décadas, así como también, el interés sobre la participación de la microbiota intestinal en la degradación de colorantes, por su parte, Chávez y col (2016) reportan metabolitos secundarios perjudiciales para la salud tras realizar un estudio de degradación del colorante allura con cepas de E. coli aisladas de un infante. Con todo ello, el deterioro de la salud humana por el consumo de alimentos procesados es evidente, la costumbre, los hábitos alimenticios y los modos de vida conlleva a un aumento del consumo de éstos productos, Barragan y col (2018) determinan que la población urbana no realiza un consumo ético, contribuyen a impedir la seguridad alimentaria.

\section{CONCLUSIONES}

Se ha diseñado y validado un instrumento de confiabilidad para medir la frecuencia de consumo alimentos procesados con rojo allura en infantes. Al mismo tiempo se conoció que la población infantil estudiada consume diariamente frituras y de forma conjunta consume de 1 a 3 veces por semana frituras, cereales y yogurt con este colorante.

\section{REFERENCIAS}

Amchova, O., H. Kotolova y J. Ruda-Kucerova, Health safety issues of synthetic food colorants, doi: 10.1016/j.yrtph.2015.09.026, Regulatory Toxicology and Pharmacology 73(3), 914-22 (2015)

Batada, A. y M.F. Jacobson, Prevalence of artificial food colors in grocery store products marketed to children, doi: 10.1177/0009922816651621, Clinical Pediatrics 55(12), 113-9 (2016)

Barragan, M. C. y D. Ayaviri, Ética del Consumo en la Gestión de la Seguridad Alimentaria en el Cantón Santo Domingo de los Colorados, Ecuador, http://dx.doi.org/10.4067/S0718-07642018000500143, Información Tecnológica 29(5),143156 (2018)

Burrows, A., Palette of our palates: A brief history of food coloring and its regulation, doi: 10.1111/j.15414337.2009.00089.x, Comprehensive Reviews in food science and food safety, 8(4), 394-408 (2009)

Ceyhan, B.M., F. Gultekin, D. K. Doguc y E. Kulac, Effects of maternally exposed coloring food additives on receptor expressions related to learning and memory in rats, doi: 10.1016/j.fct.2013.02.016, Food Chem. Toxicol. 56, 145$148(2013)$ 
Chávez, E., A.I.A. Alonso, L. Sánchez, E. Castañeda, E.V. Robles y G. Salazar, Characterization of the degradation products from the red dye 40 by enterobacteria, doi: 10.22207/JPAM.10.4.12, Journal of Pure and Applied Microbiology, 10(4), 2569-2576 (2016)

Cheung, T.T., A.F. Junghans, G.B. Dijksterhuis, F. Kroese, P. Johansson, L. Hall y D.T. De Ridder, Consumers' choiceblindness to ingredient information doi: 10.1016/j.appet.2015.09.022, Appetite, 1(106), 2-12 (2016)

Dixit S., S.K. Purshottam, S.K. Gupta, S.K. Khanna y M. Das, Usage pattern and exposure assessment of food colours in different age groups of consumers in the State of Uttar Pradesh, India, doi: 10.1080/19440040903268062, Food Addit Contam Part A Chem Anal Control Expo Risk Assess, 27(2), 181-9 (2010)

Doell, D.L., D.E. Folmer, H.S. Lee, K. M. Butts y S.E. Carberry, Exposure estimate for FD\&C color additives for the US population, doi: 10.1080/19440049.2016.1179536, Food Addit Contam Part A Chem Anal Control Expo Risk Assess, 33(5), $782-97(2016)$

EFSA Panel on Food Additives and Nutrient Sources added to Food, Refined exposure assessment for Allura Red AC (E129), EFSA Journal 13(12), 4007 (2015)

Fallico, B., E. Chiappara, E. Arena y G. Ballistreri, Assessment of the exposure to Allura Red colour from the consumption of red juice-based and red soft drinks in Italy, doi: 10.1080/19440049.2011.596166, Food Additives and Contaminants: Part A, 28(11), 1501-1515 (2011)

Feng, J., C. E. Cerniglia y H. Chen, Toxicological significance of azo dye metabolism by human intestinal microbiota, Frontiers, Bioscience (Elite Ed.) 1(4), 568-586 (2012)

Gharachorlo, M., M. Ghavami, M. Mahdiani y R. Azizinezhad, The effects of microwave frying on physicochemical properties of frying and sunflower oils, doi: 10.1007/s11746-009-1508-y, Journal of the American Oil Chemists' Society, 87, 355-360 (2010)

Gupta, V.K. y Suhas, Application of low-cost adsorbents for dye removal a review, doi: 10.1016/j.jenvman.2008.11.017, Journal of environmental management, 90(8), 2313-2342 (2009)

Nigg, J.T., K. Lewis, T. Edinger y M. Falk, Meta-analysis of attention-deficit/hyperactivity disorder or attentiondeficit/hyperactivity disorder symptoms, restriction diet, and synthetic food color additive, doi: 10.1016/j.jaac.2011.10.015, J. Am. Acad. Child. Adolesc. Psychiatry, 51(1), 86-97 (2012)

Noorafshan, A., M. Hashemi, S. Karbalay-Doust y F. Karimi, High dose Allura red, rather than the ADI dose, induces structural and behavioral changes in the medial prefron tal cortex of rats and taurine can protect it, doi: 10.1016/j.acthis.2018.07.004, Acta Histochem, 120(6), 586-594 (2018)

Martins, N., C. L. Roriz, P. Morales, L. Barros e I. C. Ferreira, Food colorants: Challenges, opportunities and current desires of agro-industries to ensure consumer expectations and regulatory practices, doi: 10.1016/j.tifs.2016.03.009, Trends in Food Science and Technology, 52, 1-15 (2016)

Monteiro, C.A., J.C. Moubarac, G. Cannon, S.W. Ng y B. Popkin, Ultra-processed products are becoming dominant in th global food system, doi: 10.1111/obr.12107, Obesity reviews, 14(2), 21-28 (2013)

Monteiro, C.A., G. Cannon, J.C. Moubarac, R.B. Levy, M.L.C. Louzada y P. Constante, The un decade of nutrition, the NOVA food classification and the trouble with ultra-processing, doi:10.1017/S1368980017000234, Public Health Nutrition 21(1), 5-17 (2017)

Pérez, C., J. Aranceta, G. Salvador y G. Varela, Métodos de frecuencia de consumo alimentario, doi: 10.14642/RENC.2015.21.sup1.5050, Rev. Nutrición comunitaria, 21(1), 45-52, (2015)

Rovina, K., S. Siddiquee y S.M. Shaarani, Extraction, analytical and advanced methods for detection of allura red ac (E129) in food and beverages products, doi: 10.3389 / fmicb.2016.00798, Frontiers in Microbiology, 7(798), 1-13 (2016)

Shimada, Ch., K. Kano, Y. F. Sasaki, I. Sato y S. Tsuda, Differential colon DNA damage induce by azo food additives between rats and mice, doi: 10.2131/jts.35.547, The Journal of Toxicological Sciences 35(4), 547-554 (2010)

Stevens, L. J., J. R. Burgess, M. A. Stochelski y T. Kuczek, Amounts of artificial food dye and added sugars in foods and sweets commonly consumed by children, doi: 10.1177/0009922814530803, Clinical Pediatrics, 1-13 (2014)

Stevens, L. J., J. R. Burgess, M. A. Stochelski y T. Kuczek, Amounts of artificial food colors in commonly consumed beverages and potential behavioral implications for consumption in children: Revisited, doi: 10.1177/0009922815581348 Clinical Pediatrics, 54(12), 1228-1230 (2015)

Vojdani, A. y C. Vojdani, Immune reactivity to food coloring, Altern Ther Health Med, 21 (Suppl. 1), 52-62 (2015)

Yamjala, K., M.S. Nainar y N.R. Ramisetti, Methods for analysis of dyes employed in food industry-A review, doi: 10.1016/j.foodchem.2015.07.085, Food Chemistry 192, 813-824 (2016) 